\title{
On the Bose-Einstein Condensation of an Ideal Gas
}

\author{
L. J. Landau and I. F. Wilde \\ Mathematics Department, Bedford College, University of London, London, UK
}

\begin{abstract}
A mathematically precise treatment is given of the well-known BoseEinstein condensation of an ideal gas in the grand canonical ensemble at fixed density. The method works equally well for any of the standard boundary conditions and it is shown that the finite volume activity converges and that in three dimensions condensation occurs for Dirichlet, Neumann, periodic, and repulsive walls.
\end{abstract}

\section{Introduction}

The phenomenon of Bose-Einstein condensation of an ideal gas is an elementary example of a phase transition. A rigorous discussion of this phenomenon within the framework of algebraic statistical mechanics was given by Araki and Woods [1] and has subsequently been developed by many authors [4-8]. It is our purpose here to present an account of Bose-Einstein condensation with particular attention given to the infinite volume limit of the grand canonical ensemble at fixed density with a careful proof of the convergence of the finite volume activities. The method we present here estimates the contribution of a collection of low lying oneparticle energy eigenstates in contrast to the customary separation of the single ground state (e.g. [7] for the case of periodic boundary conditions). As a consequence, our method allows a unified and elementary treatment of a variety of boundary conditions, although our analysis will not prove that the condensate is asymptotically in a single eigenstate. We consider the same boundary conditions as discussed by Robinson [5] and complete his analysis by allowing the finite volume activity to vary. Indeed the condensation phenomenon cannot be entirely understood in terms of grand canonical ensembles with fixed activity. (Robinson [5] shows that Dirichlet boundary conditions do not lead to condensation if the activity $z$ is fixed equal to one, but we show that if $z$ is varied with volume so as to keep the average density fixed then condensation can occur at the limit value $z=1$. The Dirichlet case with variable $z$ is also discussed in [8].) In three dimensions, condensation occurs irrespective of whether Dirichlet, Neumann, periodic or 
repulsive-wall boundary conditions are used. Moreover, they all lead to the same values of the critical temperature, activity, internal energy, and pressure in the infinite volume limit. In one or two dimensions, again they all lead to the same values of the various infinite volume limits, but there is no condensation.

The attractive-wall boundary condition considered by Robinson [5] leads to a condensation phenomenon in one, two, and three dimensions if one considers the thermodynamic quantities. However, examination of the local density demonstrates that no local condensation occurs. The thermodynamic limit of the density is larger than the density of the thermodynamic limit state and the difference between these densities is equal to the density of the condensate. Accordingly it may be argued that the attractive-wall condensation is a surface effect rather than the usual bulk phenomenon. A brief discussion of these points is given in Sect. 5 .

\section{Boundary Conditions}

We consider a free Bose gas enclosed within a cubical box of side $L$. The energy levels are determined by the eigenvalues of the one-dimensional Schrödinger equation

$$
-\frac{\hbar^{2}}{2 m} \psi^{\prime \prime}=\varepsilon \psi
$$

We consider the following boundary conditions:

$$
\begin{array}{llrl}
\text { Periodic: } & \psi(-L / 2)=\psi(L / 2), & \psi^{\prime}(-L / 2)=\psi^{\prime}(L / 2) \\
\text { Dirichlet: } & \psi(-L / 2)=0, & \psi(L / 2)=0 \\
\text { Neumann: } & \psi^{\prime}(-L / 2)=0, & \psi^{\prime}(L / 2)=0 \\
\sigma \text {-walls : } & \psi^{\prime}(-L / 2)=\sigma \psi(-L / 2), & \psi^{\prime}(L / 2)=-\sigma \psi(L / 2) .
\end{array}
$$

(These walls are repulsive if $\sigma>0$, attractive if $\sigma<0$.)

The solutions to (2.1) subject to these boundary conditions are listed in an appendix.

In two or three dimensions, the wave functions are products of the various onedimensional ones, with energy eigenvalues given by the sum of the various onedimensional energies.

We shall label the various energy eigenstates by $s$, irrespective of the dimension $(v=1,2,3)$ or the boundary conditions being used. (Of course, the values naturally assigned to $s$ will depend on $v$ and the boundary conditions.)

The local particle number density in $v$-dimensions at temperature $T$, as given by the grand canonical distribution is [4]

$$
\varrho(L, \beta, z ; x)=\sum_{s} \frac{z}{\left(e^{\beta \varepsilon(s)}-z\right)}\left|\psi_{s}(x)\right|^{2}
$$


for $x \in \mathbb{R}^{v}$, where $\beta=1 / k T$ and $z$ is the activity. The average density is $[2,3]$

$$
\begin{aligned}
\varrho(L, \beta, z) & =\frac{1}{L^{v}} \int \varrho(L, \beta, z ; x) d^{v} x \\
& =\frac{1}{L^{v}} \sum_{s} \frac{z}{\left(e^{\beta \varepsilon(s)}-z\right)} .
\end{aligned}
$$

We wish to consider the infinite volume limit, $L \rightarrow \infty$, holding the average density $\varrho(L, \beta, z)=\varrho^{*}$ fixed. The attractive-wall boundary condition will not be considered further until Sect. 5 .

Lemma 2.1. For given $L, \beta$ and $\varrho^{*}>0$, there is a unique $z_{L} \in\left(0, e^{\beta \varepsilon_{0}}\right)$ satisfying $\varrho^{*}=\varrho\left(L, \beta, z_{L}\right)$, where $\varepsilon_{0}$ is the lowest one-particle energy eigenvalue.

Proof. For any of the boundary conditions, it is easy to see that the sum in (2.3) converges and is continuous in $z$ for each fixed $L$ and $\beta$. Moreover, each summand is strictly increasing in $z$ and so, therefore, is the sum. The result follows since $\varrho(L, \beta, 0)=0$ and $\varrho(L, \beta, z) \rightarrow \infty$ as $z \rightarrow e^{\beta \varepsilon_{0}}$.

We thus have a collection $\left\{z_{L}\right\}$ in $\left(0, e^{\beta \varepsilon_{0}}\right)$, where, for each $L, z_{L}$ satisfies $\varrho^{*}=\varrho\left(L, \beta, z_{L}\right)$. We will see that $z_{L}$ converges as $L \rightarrow \infty$. By compactness, it follows that $\left\{z_{L}\right\}$ has limit points, so we only have to establish uniqueness of the limit point.

\section{Convergence of the Activity and Condensation}

In order to exhibit convergence of $z_{L}$ and explicitly illustrate the condensation phenomenon, we introduce energetically-excited particle number densities.

Definition 3.1. For $\varepsilon>0$, set

$$
\varrho_{\varepsilon}(L, \beta, z)=\frac{1}{L^{v}} \sum_{\varepsilon(s)>\varepsilon} \frac{z}{\left(e^{\beta \varepsilon(s)}-z\right)} .
$$

Theorem 3.2. Suppose $z^{*}$ is a limit point of $\left\{z_{L}\right\}$, so that there is a sequence $L_{k} \rightarrow \infty$ with $z_{L_{k}} \rightarrow z^{*}$. Then $\varrho_{\varepsilon}\left(L_{k}, \beta, z_{L_{k}}\right) \rightarrow \varrho_{\varepsilon}\left(\beta, z^{*}\right)$, where

$$
\varrho_{\varepsilon}(\beta, z)=\frac{1}{h^{v}} \int_{p^{2}>2 m \varepsilon} \frac{z}{\left(e^{\beta p^{2} / 2 m}-z\right)} d^{v} p
$$

for $0 \leqq z \leqq 1$.

Proof. We first note that whatever the boundary condition, the lowest energy $\varepsilon_{0} \downarrow 0$ as $L \rightarrow \infty$, and so $z^{*} \leqq 1$. Hence $\varrho_{\varepsilon}\left(\beta, z^{*}\right)$ is well-defined for all $\varepsilon>0$. By writing $\varepsilon(s)$ in terms of the allowed momenta, introducing the lattice spacing factors $\left(h / L_{k}\right)^{v}$, we see that (3.1) is a Riemann-sum. Since $z_{L_{k}} \rightarrow z^{*} \leqq 1$, and $\left(e^{\beta p^{2} / 2 m}-z_{L_{k}}\right)$ is continuous in $p \in \mathbb{R}^{v}$ and bounded below by $\left(e^{\beta \varepsilon}-1-\delta\right)$ for $z_{L_{k}}$ close enough to $z^{*}$, these Riemann-sums converge to the Riemann-integral

$$
\left(\frac{2}{h}\right)^{v} \int_{\substack{p^{2} \geq 2 m \varepsilon \\ p \in \mathbb{R}_{+}^{v}}} z^{*} /\left(e^{\beta p^{2} / 2 m}-z^{*}\right) d^{v} p .
$$


The result follows since $p \in \mathbb{R}^{v}, p \mapsto\left(e^{\beta p^{2} / 2 m}-z\right)$ is even.

Once we have established that $z^{*}$ is unique, we may interpret $\varrho_{\varepsilon}\left(\beta, z^{*}\right)$ as the number density of particles with energy greater than $\varepsilon$.

Definition 3.3. Let $\varrho_{e x}(\beta, z)=\sup _{\varepsilon>0} \varrho_{\varepsilon}(\beta, z)$ and let $\varrho_{0}=\varrho^{*}-\varrho_{e x}$. Then

$$
\varrho_{e x}(\beta, z)=h^{-v} \int z /\left(e^{\beta p^{2} / 2 m}-z\right) d^{v} p
$$

is the density of energetically excited particles, whereas $\varrho_{0}$ is the density of energetically unexcited particles, i.e. the density of particles which have "condensed into the ground state".

Clearly, $\varrho_{\varepsilon}\left(\beta, z^{*}\right) \leqq \varrho^{*}$ and so $\varrho_{e x}\left(\beta, z^{*}\right) \leqq \varrho^{*}$, and $\varrho_{0}\left(\beta, z^{*}\right) \geqq 0$.

Before exhibiting the condensation phenomenon in three dimensions, let us show that in one and two dimensions there is no condensation. To see this, we note that $\varrho_{e x}\left(\beta, z^{*}\right) \leqq \varrho^{*}$ implies, in particular, that $\varrho_{e x}\left(\beta, z^{*}\right)$ is finite, i.e. $z^{*} /\left(e^{\beta p^{2} / 2 m}-z^{*}\right)$ is integrable. Hence $z^{*}<1$. But then the sum for $\varrho\left(L_{k}, \beta, z_{L_{k}}\right)$ (where $z_{L_{k}} \rightarrow z^{*}$ ) converges to a Riemann-integral giving

$$
\varrho^{*}=\varrho\left(L_{k}, \beta, z_{L_{k}}\right) \rightarrow \varrho_{e x}\left(\beta, z^{*}\right) \quad \text { as } \quad L_{k} \rightarrow \infty .
$$

Thus $z^{*}$ is a solution to $\varrho^{*}=\varrho_{e x}\left(\beta, z^{*}\right)$ which is unique since $\varrho_{e x}(\beta, \cdot)$ is strictly increasing. It follows that $z_{L} \rightarrow z^{*}$ as $L \rightarrow \infty$. Moreover, $\varrho_{0}=\varrho^{*}-\varrho_{e x}=0$ for all $\beta$, and so there is no condensation.

For the remainder of this section we will consider $v=3$.

Lemma 3.4. $\varrho_{e x}(\beta, \cdot)$ is strictly increasing, and

$$
\varrho_{e x}(\beta, z) \leqq h^{-3} \int\left(e^{\beta p^{2} / 2 m}-1\right)^{-1} d^{3} p=a T^{3 / 2}
$$

with equality if and only if $z=1$, where $a$ is a constant.

Proof. Trivial, using a simple change of variable.

Definition 3.5. The critical temperature $T_{c}$ is defined by $\varrho^{*}=a T_{c}^{3 / 2}$, where $a$ is the constant of Lemma 3.4.

Theorem 3.6. If $T>T_{c}$, then any limit point $z^{*}$ of $\left\{z_{L}\right\}$ satisfies $z^{*}<1$, and $\varrho_{0}\left(\beta, z^{*}\right)=0$. Conversely, if there exists a limit point $z^{*}<1$, then $T>T_{c}$ and so all limit points lie in $(0,1)$.

Proof. Let $z^{*}$ be a limit point of $\left\{z_{L}\right\}$, and suppose $T>T_{c}$. Then

$$
\varrho_{e x}\left(\beta, z^{*}\right) \leqq \varrho^{*}=a T_{c}^{3 / 2}<a T^{3 / 2}=\varrho_{e x}(\beta, 1) .
$$

Hence, by Lemma $3.4, z^{*}<1$. It follows that

$$
\varrho^{*}=L_{k}^{-3} \sum_{s} z_{L_{k}} /\left(e^{\beta \varepsilon(s)}-z_{L_{k}}\right) \rightarrow h^{-3} \int \frac{z^{*}}{e^{\beta p^{2} / 2 m}-z^{*}} d^{3} p
$$

for some sequence $z_{L_{k}} \rightarrow z^{*}$. That is, $\varrho^{*}=\varrho_{e x}\left(\beta, z^{*}\right)$, and so $\varrho_{0}\left(\beta, z^{*}\right)=0$.

Conversely, if $z^{*}<1$ is a limit point, we can reverse this last argument to conclude that $\varrho^{*}=\varrho_{e x}\left(\beta, z^{*}\right)$ and thus, using Lemma 3.4,

$$
a T_{c}^{3 / 2}=\varrho^{*}=\varrho_{e x}\left(\beta, z^{*}\right)<\varrho_{e x}(\beta, 1)=a T^{3 / 2} .
$$

Hence $T_{c}<T$ as required. 
Corollary 3.7. If $T>T_{c}$, then $z_{L} \rightarrow z^{*}$ as $L \rightarrow \infty$, for some $z^{*}<1$.

Proof. Suppose $z^{*}$ and $z^{* *}$ are limit points of $\left\{z_{L}\right\}$. Then, if $T>T_{c}$, we conclude that both $z^{*}<1$ and $z^{* *}<1$. Moreover,

$$
\varrho_{e x}\left(\beta, z^{*}\right)=\varrho^{*}=\varrho_{e x}\left(\beta, z^{* *}\right) .
$$

By Lemma 3.4, we deduce that $z^{*}=z^{* *}$.

We see then, irrespective of the boundary conditions, that above $T_{c}$ the infinite volume activity $z^{*}$ is less than unity, and, in fact, is the unique solution to $\varrho^{*}=\varrho_{e x}\left(\beta, z^{*}\right)$. Moreover, $\varrho_{0}\left(\beta, z^{*}\right)=0$ and so there is no Bose-Einstein condensation.

Our next result shows that below $T_{c}$ there is condensation (in 3 dimensions).

Theorem 3.8. If $T \leqq T_{c}$, then $z_{L} \rightarrow 1$ as $L \rightarrow \infty$, and $\varrho_{0}(\beta, 1)=\varrho^{*}\left(1-\left(T / T_{c}\right)^{3 / 2}\right)$.

Proof. Since $T \leqq T_{c}$, it follows from Theorem 3.6 that $\left\{z_{L}\right\}$ can have no limit points smaller than 1 . Hence $z_{L} \rightarrow 1$ as $L \rightarrow \infty$, and we have

$$
\varrho_{0}(\beta, 1)=\varrho^{*}-\varrho_{e x}(\beta, 1)=\varrho^{*}-a T^{3 / 2} .
$$

The result follows since $a=\varrho^{*} T_{c}^{-3 / 2}$.

We have thus exhibited the Bose-Einstein condensation, $\varrho_{0}>0$, below $T_{c}$, irrespective of the boundary conditions. The phase transition which occurs at $T=T_{c}$ manifests itself in the non-analytic behaviour of the function $z(\varrho, T)$. This gives rise to different analytic expressions for the thermodynamic functions as $T$ passes through $T_{c}$ and to different phases.

\section{The Internal Energy and Pressure}

In this section, we wish to establish the existence of the specific internal energy and the pressure in the thermodynamic limit.

The internal energy per unit volume and the pressure are given, respectively, by $[2,3]$

$$
u\left(L, \beta, z_{L}\right)=L^{-v} \sum_{s} \frac{z_{L} \varepsilon(s)}{\left(e^{\beta \varepsilon(s)}-z_{L}\right)}
$$

and

$$
p\left(L, \beta, z_{L}\right)=\frac{-1}{\beta L^{v}} \sum_{s} \log \left(1-z_{L} e^{-\beta \varepsilon(s)}\right)
$$

where $z_{L}$ is given by $\varrho^{*}=\varrho\left(L, \beta, z_{L}\right)$.

\section{Theorem 4.1.}

$$
\lim _{L \rightarrow \infty} u\left(L, \beta, z_{L}\right)=h^{-v} \int \frac{p^{2}}{2 m} \frac{z^{*}}{\left(e^{\beta p^{2} / 2 m}-z^{*}\right)} d^{v} p
$$


Proof. Set

$$
u_{\varepsilon}\left(L, \beta, z_{L}\right)=L^{-v} \sum_{\varepsilon(s)>\varepsilon} \frac{z_{L} \varepsilon(s)}{\left(e^{\beta \varepsilon(s)}-z_{L}\right)} .
$$

Then, as in Theorem 3.2,

$$
u_{\varepsilon}\left(L, \beta, z_{L}\right) \rightarrow u_{\varepsilon}\left(\beta, z^{*}\right)=\frac{1}{h^{v}} \int_{p^{2}>2 m \varepsilon} \frac{p^{2}}{2 m} \frac{z^{*}}{\left(e^{\beta p^{2} / 2 m}-z^{*}\right)} d^{v} p .
$$

Now,

$$
\begin{aligned}
0 & \leqq u\left(L, \beta, z_{L}\right)-u_{\varepsilon}\left(L, \beta, z_{L}\right)=L^{-v} \sum_{\varepsilon(s) \leqq \varepsilon} \frac{z_{L} \varepsilon(s)}{\left(e^{\beta \varepsilon(s)}-z_{L}\right)} \\
& \leqq \varepsilon L^{-v} \sum_{s} \frac{z_{L}}{e^{\beta \varepsilon(s)}-z_{L}}=\varepsilon Q^{*}
\end{aligned}
$$

which goes to zero as $\varepsilon \rightarrow 0$. It follows that

$$
\lim _{L \rightarrow \infty} u\left(L, \beta, z_{L}\right)=\lim _{\varepsilon \downarrow 0} u_{\varepsilon}\left(\beta, z^{*}\right)=\frac{1}{h^{v}} \int \frac{p^{2}}{2 m} \frac{z^{*}}{\left(e^{\beta p^{2} / 2 m}-z^{*}\right)} d^{v} p .
$$

\section{Theorem 4.2.}

$$
\lim _{L \rightarrow \infty} p(L, \beta, z)=-\frac{1}{\beta h^{v}} \int \log \left(1-z^{*} e^{-\beta p^{2} / 2 m}\right) d^{v} p
$$

where $z^{*}=\lim z_{L}$.

Proof. Set

$$
p_{\varepsilon}\left(L, \beta, z_{L}\right)=-\frac{1}{\beta L^{v}} \sum_{\varepsilon(s)>\varepsilon} \log \left(1-z_{L} e^{-\beta \varepsilon(s)}\right) .
$$

Then, as in Theorem 3.2,

$$
p_{\varepsilon}\left(L, \beta, z_{L}\right) \rightarrow p_{\varepsilon}\left(\beta, z^{*}\right)=-\frac{1}{\beta h^{v}} \int_{p^{2}>2 m \varepsilon} \log \left(1-z^{*} e^{-\beta p^{2} / 2 m}\right) d^{v} p .
$$

Now,

$$
p\left(L, \beta, z_{L}\right)-p_{\varepsilon}\left(L, \beta, z_{L}\right)=\frac{1}{\beta L^{v}} \sum_{\varepsilon(s) \leqq \varepsilon} \log \frac{1}{\left(1-z_{L} e^{-\beta \varepsilon(s)}\right)} .
$$

Using the inequality $0 \leqq \sqrt{x} \log \frac{1}{x} \leqq \frac{2}{e}$ for $0 \leqq x \leqq 1$, we obtain

$$
\begin{aligned}
0 & \leqq p\left(L, \beta, z_{L}\right)-p_{\varepsilon}\left(L, \beta, z_{L}\right) \leqq \frac{2}{e \beta L^{v}} \sum_{\varepsilon(s) \leqq \varepsilon} \frac{1}{\left(1-z_{L} e^{-\beta \varepsilon(s)}\right)^{1 / 2}} \\
& \leqq \frac{2}{e \beta}\left\{\frac{1}{L^{v}} \sum_{\varepsilon(s) \leqq \varepsilon} \frac{1}{\left(1-z_{L} e^{-\beta \varepsilon(s)}\right)}\right\}^{1 / 2}\left\{\frac{1}{L^{v}} \sum_{\varepsilon(s) \leqq \varepsilon} 1\right\}^{1 / 2}
\end{aligned}
$$


using Schwarz' inequality,

$$
\leqq \frac{2}{\left(e \beta z_{L} e^{-\beta \varepsilon}\right)^{1 / 2}}\left\{\frac{1}{L^{v}} \sum_{\varepsilon(s) \leqq \varepsilon} \frac{z_{L} e^{-\beta \varepsilon(s)}}{\left(1-z_{L} e^{-\beta \varepsilon(s)}\right)}\right\}^{1 / 2}\left\{\frac{1}{L^{v}} \sum_{\varepsilon(s) \leqq \varepsilon} 1\right\}^{1 / 2} .
$$

The expression in the first bracket is bounded by $\varrho^{*}$, and that in the second bracket is simply a Riemann sum approximation which converges to $h^{-v} \int_{p^{2} \leqq 2 m \varepsilon} d^{v} p$, which tends to zero as $\varepsilon \downarrow 0$. It follows that

$$
\begin{aligned}
\lim _{L \rightarrow \infty} p\left(L, \beta, z_{L}\right) & =\lim _{\varepsilon \downarrow 0} p_{\varepsilon}\left(\beta, z^{*}\right) \\
& =-\frac{1}{\beta h^{v}} \int \log \left(1-z^{*} e^{-\beta p^{2} / 2 m}\right) d^{v} p .
\end{aligned}
$$

We observe that the infinite volume limits are independent of the boundary conditions, but that they involve $z^{*}$; i.e. they exhibit non-analytic behaviour as $T$ passes through $T_{c}$.

\section{Attractive-Wall Boundary Conditions and the Local Density}

The attractive-wall boundary condition $(\sigma<0)$ may be treated in a similar way. A condensation phenomenon does occur in one, two, and three dimensions, but this could be viewed as a surface effect as can be seen for example by considering the local density.

As before, the finite volume activity $z_{L}$ is the unique solution to $\varrho^{*}=\varrho(L, \beta, z)$ satisfying $0 \leqq z_{L}<e^{\beta \varepsilon_{0}}$. Since the ground state energy $\varepsilon_{0} \rightarrow-\frac{\hbar^{2}}{2 m} v \sigma^{2}$ as $L \rightarrow \infty$, any limit point of $\left\{z_{L}\right\}$ lies in $\left[0, e^{-\beta \hbar^{2} v \sigma^{2} / 2 m}\right]$.

We set

$$
\phi(T, \sigma)=\varrho_{e x}\left(\beta, e^{-\beta \hbar^{2} v \sigma^{2} / 2 m}\right)=\frac{1}{h^{v}} \int \frac{e^{-\beta \hbar^{2} v \sigma^{2} / 2 m}}{e^{\beta p^{2} / 2 m}-e^{-\beta \hbar^{2} v \sigma^{2} / 2 m}} d^{v} p .
$$

The critical temperature $T_{c}$ is now defined by $\varrho^{*}=\phi\left(T_{c}, \sigma\right)$. $T_{c}$ is well-defined since $\phi(\cdot, \sigma)$ is strictly increasing, and $\phi(T, \sigma) \rightarrow 0$ as $T \rightarrow 0$, and $\phi(T, \sigma) \rightarrow \infty$ as $T \rightarrow \infty$. An analysis similar to that of Sects. 3 and 4 leads to

Theorem 5.1. In $v$-dimensions, $v=1,2,3$, the free Bose gas subject to attractive-wall boundary conditions exhibits a condensation phenomenon $\left(\varrho_{0} \neq 0\right)$ in the thermodynamic limit. The critical temperature is given by $\varrho^{*}=\phi\left(T_{c}, \sigma\right)$. For $T>T_{c}$, the activities $z_{L}$ converge, as $L \rightarrow \infty$, to $z^{*}$, the unique solution to $\varrho^{*}=\varrho_{e x}\left(\beta, z^{*}\right)$ and $\varrho_{0}\left(\beta, z^{*}\right)=0$. If $T \leqq T_{c}, z_{L}$ converges to $e^{-\beta \hbar^{2} v \sigma^{2} / 2 m}$ as $L \rightarrow \infty$. In this case,

$$
\varrho_{0}\left(\beta, e^{-\beta \hbar^{2} \vee \sigma^{2} / 2 m}\right)=\phi\left(T_{c}, \sigma\right)-\phi(T, \sigma) \geqq 0 .
$$




\section{Theorem 5.2.}

$$
\lim _{L \rightarrow \infty} u\left(L, \beta, z_{L}\right)=\frac{1}{h^{v}} \int \frac{p^{2}}{2 m} \frac{z^{*}}{e^{\beta p^{2} / 2 m}-z^{*}} d^{v} p-\frac{\hbar v \sigma^{2}}{2 m} \varrho_{0}\left(\beta, z^{*}\right)
$$

where $z^{*}=\lim _{L \rightarrow \infty} z_{L}$.

Note that $-\frac{\hbar^{2} v \sigma^{2}}{2 m} \varrho_{0}\left(\beta, z^{*}\right)$ is just the binding energy per particle due to the states bound to the walls. The energy is actually "located at infinity".

\section{Theorem 5.3.}

$$
\lim _{L \rightarrow \infty} p\left(L, \beta, z_{L}\right)=-\frac{1}{\beta h^{v}} \int \log \left(1-z^{*} e^{-\beta p^{2} / 2 m}\right) d^{v} p
$$

where $z^{*}=\lim _{L \rightarrow \infty} z_{L}$.

If one uses the explicit formulae for the one-particle wave functions (see the appendix) then the limit as $L \rightarrow \infty$ of the local density (Eq. 2.2) may be computed. The limit density is translation invariant and equals the global density in the case of periodic, Dirichlet, Neumann and repulsive-wall boundary conditions, but for attractive-wall boundary conditions the local density is equal to $\varrho_{e x}$.

Theorem 5.4. For periodic, Dirichlet, Neumann and repulsive-wall $(\sigma>0)$ boundary conditions, the local density $\varrho\left(L, \beta, z_{L} ; x\right)$ converges as $L \rightarrow \infty$, to the global density $\varrho^{*}$. For attractive-wall $(\sigma<0)$ boundary conditions, $\varrho\left(L, \beta, z_{L} ; x\right)$ converges to $\varrho_{e x}\left(\beta, z^{*}\right)$.

\section{Appendix}

The one-particle energy levels are determined by the eigenvalues of the onedimensional Schrödinger equation

$$
-\frac{\hbar^{2}}{2 m} \psi^{\prime \prime}=\varepsilon \psi
$$

with the appropriate boundary condition (Sect. 2).

The eigenvectors are either even $(e)$ or odd $(o)$ with respect to $x \rightarrow-x$, and are labeled by the integer $n$ :

$$
\begin{array}{ll}
\psi_{n}^{(o)}(x)=\left(\frac{2}{L}\right)^{1 / 2} \frac{\sin \frac{1}{\hbar} p_{n}^{(o)} x}{\sqrt{\left(1+b_{n}^{(o)}\right)}}, \quad \begin{array}{l}
n=1,2, \ldots \\
(\text { In the case } \sigma<0, n=0 \text { is also allowed.) }
\end{array} \\
\psi_{n}^{(e)}(x)=\left(\frac{2}{L}\right)^{1 / 2} \frac{\cos \frac{1}{\hbar} p_{n}^{(e)} x}{\sqrt{\left(1+b_{n}^{(e)}\right)}}, \quad n=0,1,2, \ldots
\end{array}
$$


where

Dirichlet $p_{n}^{(o)}=\frac{h}{L} n, \quad b_{n}^{(o)}=0, \quad p_{n}^{(e)}=\frac{h}{L}\left(n+\frac{1}{2}\right), \quad b_{n}^{(e)}=0$

Neumann $p_{n}^{(o)}=\frac{h}{L}\left(n-\frac{1}{2}\right), \quad b_{n}^{(o)}=0, \quad p_{n}^{(e)}=\frac{h}{L} n, \quad b_{n}^{(e)}= \begin{cases}0 & n \neq 0 \\ 1 & n=0\end{cases}$

Periodic $\quad p_{n}^{(o)}=\frac{h}{L} n, \quad b_{n}^{(o)}=0, \quad p_{n}^{(e)}=\frac{h}{L} n, \quad b_{n}^{(e)}= \begin{cases}0 & n \neq 0 \\ 1 & n=0\end{cases}$

$\sigma>0 \quad p_{n}^{(o)}=\frac{h}{L} a_{n}^{(o)}, \quad b_{n}^{(o)}=-\frac{\sin 2 \pi a_{n}^{(o)}}{2 \pi a_{n}^{(o)}}, \quad n-\frac{1}{2}<a_{n}^{(o)}<n, \quad a_{n}^{(o)} \underset{L \rightarrow \infty}{\nearrow} n$

$$
p_{n}^{(e)}=\frac{h}{L} a_{n}^{(e)}, \quad b_{n}^{(e)}=\frac{\sin 2 \pi a_{n}^{(e)}}{2 \pi a_{n}^{(e)}}, \quad n<a_{n}^{(e)}<n+\frac{1}{2}, \quad a_{n}^{(e)} \underset{L \rightarrow \infty}{\nearrow} n+\frac{1}{2}
$$

$\sigma<0 \quad p_{n}^{(o)}=\frac{h}{L} a_{n}^{(o)}, \quad b_{n}^{(o)}=-\frac{\sin 2 \pi a_{n}^{(o)}}{2 \pi a_{n}^{(o)}}, \quad n<a_{n}^{(o)}<n+\frac{1}{2}, \quad a_{n}^{(o)} \underset{L \rightarrow \infty}{\searrow} n$

$$
p_{n}^{(e)}=\frac{h}{L} a_{n}^{(e)}, \quad b_{n}^{(e)}=\frac{\sin 2 \pi a_{n}^{(e)}}{2 \pi a_{n}^{(e)}}, \quad n+\frac{1}{2}<a_{n}^{(e)}<n+1, \quad a_{n}^{(e)} \underset{L \rightarrow \infty}{\searrow} n+\frac{1}{2} .
$$

Furthermore in the case $\sigma<0$ there is an even and an odd bound state (if $\left.L>\frac{2}{|\sigma|}\right)$ with $\varepsilon=-\sigma^{2} \frac{\hbar^{2}}{2 m} a^{2}, a^{(o)} \underset{L \rightarrow \infty}{\nearrow} 1, a^{(e)} \underset{L \rightarrow \infty}{\searrow} 1$, with wave functions

$$
\begin{gathered}
\psi^{(o)}(x)=\left(\frac{2}{L}\right)^{1 / 2} \sinh \left(\sigma a^{(o)} x\right)\left(\frac{\left.\sinh L|\sigma| a^{(o)}\right)}{L|\sigma| a^{(o)}}-1\right)^{-1 / 2} \\
\psi^{(e)}(x)=\left(\frac{2}{L}\right)^{1 / 2} \cosh \left(\sigma a^{(e)} x\right)\left(\frac{\sinh \left(L|\sigma| a^{(e)}\right)}{L|\sigma| a^{(e)}}+1\right)^{-1 / 2}
\end{gathered}
$$

\section{References}

1. Araki, H., Woods, E.J.: J. Math. Phys. 4, 637-662 (1963)

2. Huang, K.: Statistical mechanics. London: Pergamon 1958

3. Jost, R.: Quantenmechanik II. ETH Zürich, Verlag der Fachvereine an der ETH/Z, 1973

4. Lewis, J., Pule, J.V.: Comm. Math. Phys. 36, 1-18 (1974)

5. Robinson, D.W.: Comm. Math. Phys. 50, 53-59 (1976)

6. Rocca, F., Sirugue, M., Testard, D.: Comm. Math. Phys. 19, 119-141 (1970)

7. Cannon, J.: Comm. Math. Phys. 29, 89-104 (1973)

8. Bratteli, O., Robinson, D.W.: Chapter 5 of draft version of forthcoming book

Communicated by E. Lieb

Received May 7, 1979; in revised form May 29, 1979 
